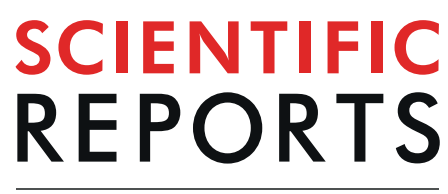

natureresearch

Check for updates

\title{
Germline HOXB13 mutations \\ p.G84E and p.R217C do not confer an increased breast cancer risk
}

Jingjing Liu ${ }^{1,2}$, Wendy J. C. Prager - van der Smissen ${ }^{1}$, J. Margriet Collée ${ }^{3}$, Manjeet K. Bolla ${ }^{4}$, Qin Wang ${ }^{4}$, Kyriaki Michailidou ${ }^{4,5,6}$, Joe Dennis ${ }^{4}$, Thomas U. Ahearn 7 , Kristiina Aittomäki ${ }^{8}$, Christine B. Ambrosone ${ }^{9}$, Irene L. Andrulis ${ }^{10,11}$, Hoda AntonCulver ${ }^{12}$, Natalia N. Antonenkova ${ }^{13}$, Volker Arndt ${ }^{14}$, Norbert Arnold ${ }^{15,16}$, Kristan J. Aronson ${ }^{17}$, Annelie Augustinsson ${ }^{18}$, Päivi Auvinen ${ }^{19,20,21}$, Heiko Becher ${ }^{22,23}$, Matthias W. Beckmann ${ }^{24}$, Sabine Behrens ${ }^{25}$, Marina Bermisheva ${ }^{26}$, Leslie Bernstein ${ }^{27}$, Natalia V. Bogdanova ${ }^{13,28,29}$, Nadja Bogdanova-Markov ${ }^{30}$, Stig E. Bojesen ${ }^{31,32,33}$, Hiltrud Brauch ${ }^{34,35,36}$, Hermann Brenner ${ }^{14,36,37}$, Ignacio Briceno ${ }^{38,39}$, Sara Y. Brucker ${ }^{40}$, Thomas Brüning ${ }^{41}$, Barbara Burwinkel ${ }^{42,43}$, Qiuyin Cai ${ }^{44}$, Hui Caii ${ }^{44}$, Daniele Campa ${ }^{25,45}$, Federico Canzian ${ }^{46}$, Jose E. Castelao ${ }^{47}$, Jenny Chang-Claude ${ }^{25,48}$, Stephen J. Chanock ${ }^{7}$, Ji-Yeob Choi ${ }^{49,50}$, Melissa Christiaens ${ }^{51}$, Christine L. Clarke ${ }^{52}$, NBCS Collaborators*, Fergus J. Couch ${ }^{63}$, Kamila Czene ${ }^{64}$, Mary B. Daly ${ }^{65}$, Peter Devilee ${ }^{66,67}$, Isabel dos-SantosSilva $^{68}$, Miriam Dwek ${ }^{69}$, Diana M. Eccles ${ }^{70}$, A. Heather Eliassen ${ }^{71,72}$, Peter A. Fasching ${ }^{24,73}$, Jonine Figueroa ${ }^{7,74,75}$, Henrik Flyger ${ }^{76}$, Lin Fritschi ${ }^{77}$, Manuela Gago-Dominguez ${ }^{78,79}$, Susan M. Gapstur ${ }^{80}$, Montserrat García-Closas ${ }^{7}$, José A. García-Sáenz ${ }^{81}$, Mia M. Gaudet ${ }^{80}$, Graham G. Giles ${ }^{82,83,84}$, Mark S. Goldberg ${ }^{85,86}$, David E. Goldgar ${ }^{87}$, Pascal Guénel ${ }^{88}$, Christopher A. Haiman ${ }^{89}$, Niclas Håkansson ${ }^{90}$, Per Hall ${ }^{64,91}$, Patricia A. Harrington ${ }^{92}$, Steven N. Hart ${ }^{93}$, Mikael Hartman ${ }^{94,95}$, Peter Hillemanns ${ }^{29}$, John L. Hopper ${ }^{83}$, MingFeng Hou ${ }^{96}$, David J. Hunter ${ }^{72,97,98}$, Dezheng Huo ${ }^{99}$, ABCTB Investigators*, Hidemi Ito ${ }^{100,101}$, Motoki Iwasaki ${ }^{102}$, Milena Jakimovska ${ }^{103}$, Anna Jakubowska ${ }^{104,105}$, Esther M. John ${ }^{106}$, Rudolf Kaaks ${ }^{25}$, Daehee Kang ${ }^{49,50,107}$, Renske Keeman ${ }^{108}$, Elza Khusnutdinova ${ }^{26,109}$, Sung-Won Kim ${ }^{110}$, Peter Kraft ${ }^{72,97}$, Vessela N. Kristensen ${ }^{111}$, Allison W. Kurian ${ }^{106,112,}$ Loic Le Marchand ${ }^{113}$, Jingmei Li ${ }^{114}$, Annika Lindblom ${ }^{115,116}$, Artitaya Lophatananon ${ }^{117}$, Robert N. Luben ${ }^{118}$, Jan Lubiński ${ }^{104}$, Arto Mannermaa ${ }^{21,119,120}$, Mehdi Manoochehri ${ }^{121}$, Siranoush Manoukian ${ }^{122}$, Sara Margolin ${ }^{91,123}$, Shivaani Mariapun ${ }^{124}$, Keitaro Matsuo ${ }^{100,101}$, Tabea Maurer ${ }^{48}$, Dimitrios Mavroudis ${ }^{125}$, Alfons Meind ${ }^{126}$, Usha Menon ${ }^{127}$, Roger L. Milne ${ }^{82,83,84}$, Kenneth Muir ${ }^{117}$, Anna Marie Mulligan ${ }^{128,129}$, Susan L. Neuhausen ${ }^{27}$, Heli Nevanlinna ${ }^{130}$, Kenneth Offit ${ }^{131,132}$, Olufunmilayo I. Olopade ${ }^{99}$, Janet E. Olson ${ }^{93}$, Håkan Olsson ${ }^{18}$, Nick Orr ${ }^{133,134}$, Sue K. Park ${ }^{49,50,107}$, Paolo Peterlongo ${ }^{135}$, Julian Peto ${ }^{68}$, Dijana Plaseska-Karanfilska ${ }^{103}$, Nadege Presneau ${ }^{69}$, Brigitte Rack ${ }^{136}$, Rohini RauMurthy ${ }^{132}$, Gad Rennert ${ }^{137}$, Hedy S. Rennert ${ }^{137}$, Valerie Rhenius ${ }^{92}$, Atocha Romero ${ }^{138}$, Matthias Ruebner ${ }^{139}$, Emmanouil Saloustros ${ }^{140}$, Rita K. Schmutzler ${ }^{141,142,143}$, Andreas Schneeweiss ${ }^{43,144}$, Christopher Scott ${ }^{93}$, Mitul Shah ${ }^{92}$, Chen-Yang Shen ${ }^{145,146}$, Xiao-Ou Shu ${ }^{44}$, Jacques Simard ${ }^{147}$, Christof Sohn ${ }^{144}$, Melissa C. Southey $82,84,148$, John J. Spinelli ${ }^{149,150}$, Rulla M. Tamimi ${ }^{71,72,97}$, William J. Tapper ${ }^{70}$, Soo H. Teo ${ }^{151,152}$, Mary Beth Terry ${ }^{153}$, Diana Torres ${ }^{38,121}$, Thérèse Truong ${ }^{88}$, Michael Untch ${ }^{154}$, Celine M. Vachon ${ }^{155}$, Christi J. van Asperen ${ }^{156}$, Alicja Wolk ${ }^{90,157}$, Taiki Yamaji ${ }^{102}$, Wei Zheng ${ }^{44}$, Argyrios Ziogas ${ }^{12}$, Elad Ziv ${ }^{158}$, Gabriela Torres-Mejía ${ }^{159}$, Thilo Dörk ${ }^{29}$, Anthony J. Swerdlow ${ }^{160,161}$, Ute Hamann ${ }^{121}$, Marjanka K. Schmidt ${ }^{108,162}$, Alison M. Dunning ${ }^{92}$, Paul D. P. Pharoah ${ }^{4,92}$, Douglas F. Easton ${ }^{4,92}$, Maartje J. Hooning ${ }^{1}$, John W. M. Martens ${ }^{1}$ \& Antoinette Hollestelle ${ }^{1 \bowtie}$ 
${ }^{1}$ Department of Medical Oncology, Family Cancer Clinic, Erasmus MC Cancer Institute, Rotterdam, The Netherlands. ${ }^{2}$ Institute of Medical and Pharmaceutical Sciences, Zhengzhou University, Zhengzhou, China. ${ }^{3}$ Department of Clinical Genetics, Erasmus University Medical Center, Rotterdam, The Netherlands. ${ }^{4}$ Centre for Cancer Genetic Epidemiology, Department of Public Health and Primary Care, University of Cambridge, Cambridge, UK. ${ }^{5}$ Biostatistics Unit, The Cyprus Institute of Neurology \& Genetics, Nicosia, Cyprus. ${ }^{6}$ Cyprus School of Molecular Medicine, The Cyprus Institute of Neurology \& Genetics, Nicosia, Cyprus. ${ }^{7}$ Division of Cancer Epidemiology and Genetics, National Cancer Institute, National Institutes of Health, Department of Health and Human Services, Bethesda, MD, USA. ${ }^{8}$ Department of Clinical Genetics, Helsinki University Hospital, University of Helsinki, Helsinki, Finland. ${ }^{9}$ Roswell Park Cancer Institute, Buffalo, NY, USA. ${ }^{10}$ Fred A. Litwin Center for Cancer Genetics, LunenfeldTanenbaum Research Institute of Mount Sinai Hospital, Toronto, ON, Canada. ${ }^{11}$ Department of Molecular Genetics, University of Toronto, Toronto, ON, Canada. ${ }^{12}$ Department of Epidemiology, Genetic Epidemiology Research Institute, University of California Irvine, Irvine, CA, USA. ${ }^{13} \mathrm{~N}$.N. Alexandrov Research Institute of Oncology and Medical Radiology, Minsk, Belarus. ${ }^{14}$ Division of Clinical Epidemiology and Aging Research, German Cancer Research Center (DKFZ), Heidelberg, Germany. ${ }^{15}$ Department of Gynaecology and Obstetrics, University Hospital of Schleswig-Holstein, Campus Kiel, Christian-Albrechts University Kiel, Kiel, Germany. ${ }^{16}$ Institute of Clinical Molecular Biology, University Hospital of Schleswig-Holstein, Campus Kiel, Christian-Albrechts University Kiel, Kiel, Germany. ${ }^{17}$ Department of Public Health Sciences, and Cancer Research Institute, Queen's University, Kingston, ON, Canada. ${ }^{18}$ Department of Cancer Epidemiology, Clinical Sciences, Lund University, Lund, Sweden. ${ }^{19}$ Cancer Center, Kuopio University Hospital, Kuopio, Finland. ${ }^{20}$ Institute of Clinical Medicine, Oncology, University of Eastern Finland, Kuopio, Finland. ${ }^{21}$ Translational Cancer Research Area, University of Eastern Finland, Kuopio, Finland. ${ }^{22}$ Institute of Medical Biometry and Epidemiology, University Medical Center Hamburg-Eppendorf, Hamburg, Germany. ${ }^{23}$ Institute of Biometry and Clinical Epidemiology, Charité -Universitätsmedizin Berlin, Berlin, Germany. ${ }^{24}$ Department of Gynecology and Obstetrics, Comprehensive Cancer Center ER-EMN, University Hospital Erlangen, FriedrichAlexander-University Erlangen-Nuremberg, Erlangen, Germany. ${ }^{25}$ Division of Cancer Epidemiology, German Cancer Research Center (DKFZ), Heidelberg, Germany. ${ }^{26}$ Institute of Biochemistry and Genetics, Ufa Federal Research Centre of the Russian Academy of Sciences, Ufa, Russia. ${ }^{27}$ Department of Population Sciences, Beckman Research Institute of City of Hope, Duarte, CA, USA. ${ }^{28}$ Department of Radiation Oncology, Hannover Medical School, Hannover, Germany. ${ }^{29}$ Gynaecology Research Unit, Hannover Medical School, Hannover, Germany. ${ }^{30}$ Institute of Human Genetics, University of Münster, Münster, Germany. ${ }^{31}$ Copenhagen General Population Study, Herlev and Gentofte Hospital, Copenhagen University Hospital, Herlev, Denmark. ${ }^{32}$ Department of Clinical Biochemistry, Herlev and Gentofte Hospital, Copenhagen University Hospital, Herlev, Denmark. ${ }^{33}$ Faculty of Health and Medical Sciences, University of Copenhagen, Copenhagen, Denmark. ${ }^{34} \mathrm{Dr}$. Margarete Fischer-Bosch-Institute of Clinical Pharmacology, Stuttgart, Germany. ${ }^{35} \mathrm{FFIT}$-Cluster of Excellence, University of Tübingen, Tübingen, Germany. ${ }^{36}$ German Cancer Consortium (DKTK), German Cancer Research Center (DKFZ), Partner Site Tübingen, Tübingen, Germany. ${ }^{37}$ Division of Preventive Oncology, German Cancer Research Center (DKFZ) and National Center for Tumor Diseases (NCT), Heidelberg, Germany. ${ }^{38}$ Institute of Human Genetics, Pontificia Universidad Javeriana, Bogota, Colombia. ${ }^{39}$ Medical Faculty, Universidad de La Sabana, Bogota, Colombia. ${ }^{40}$ Department of Gynecology and Obstetrics, University of Tübingen, Tübingen, Germany. ${ }^{41}$ Institute for Prevention and Occupational Medicine of the German Social Accident Insurance, Institute of the Ruhr University Bochum (IPA), Bochum, Germany. ${ }^{42}$ Molecular Epidemiology Group, C080, German Cancer Research Center (DKFZ), Heidelberg, Germany. ${ }^{43}$ Molecular Biology of Breast Cancer, University Womens Clinic Heidelberg, University of Heidelberg, Heidelberg, Germany. ${ }^{44}$ Division of Epidemiology, Department of Medicine, Vanderbilt Epidemiology Center, Vanderbilt-Ingram Cancer Center, Vanderbilt University School of Medicine, Nashville, TN, USA. ${ }^{45}$ Department of Biology, University of Pisa, Pisa, Italy. ${ }^{46}$ Genomic Epidemiology Group, German Cancer Research Center (DKFZ), Heidelberg, Germany. ${ }^{47}$ Oncology and Genetics Unit, Instituto de Investigacion Sanitaria Galicia Sur (IISGS), Xerencia de Xestion Integrada de Vigo-SERGAS, Vigo, Spain. ${ }^{48} \mathrm{Cancer}$ Epidemiology Group, University Cancer Center Hamburg (UCCH), University Medical Center Hamburg-Eppendorf, Hamburg, Germany. ${ }^{49}$ Department of Biomedical Sciences, Seoul National University Graduate School, Seoul, Korea. ${ }^{50}$ Cancer Research Institute, Seoul National University, Seoul, Korea. ${ }^{51}$ Leuven Multidisciplinary Breast Center, Department of Oncology, Leuven Cancer Institute, University Hospitals Leuven, Leuven, Belgium. ${ }^{52}$ Westmead Institute for Medical Research, University of Sydney, Sydney, New South Wales, Australia. ${ }^{63}$ Department of Laboratory Medicine and Pathology, Mayo Clinic, Rochester, MN, USA. ${ }^{64}$ Department of Medical Epidemiology and Biostatistics, Karolinska Institutet, Stockholm, Sweden. ${ }^{65}$ Department of Clinical Genetics, Fox Chase Cancer Center, Philadelphia, PA, USA. ${ }^{66}$ Department of Pathology, Leiden University Medical Center, Leiden, The Netherlands. ${ }^{67}$ Department of Human Genetics, Leiden University Medical Center, Leiden, The Netherlands. ${ }^{68}$ Department of NonCommunicable Disease Epidemiology, London School of Hygiene and Tropical Medicine, London, UK. ${ }^{69}$ School of Life Sciences, University of Westminster, London, UK. ${ }^{70}$ Faculty of Medicine, University of Southampton, Southampton, UK. ${ }^{71}$ Channing Division of Network Medicine, Department of Medicine, Brigham and Women's Hospital and Harvard Medical School, Boston, MA, USA. ${ }^{72}$ Department of Epidemiology, Harvard T.H. Chan School of Public Health, Boston, MA, USA. ${ }^{73}$ David Geffen School of Medicine, Department of Medicine Division of Hematology and Oncology, University of California at Los Angeles, Los Angeles, CA, USA. ${ }^{74}$ Usher Institute of Population Health Sciences and Informatics, The University of Edinburgh, Edinburgh, UK. ${ }^{75}$ Cancer Research UK Edinburgh Centre, The University of Edinburgh, Edinburgh, UK. ${ }^{76}$ Department of Breast Surgery, Herlev and Gentofte Hospital, Copenhagen University Hospital, Herlev, Denmark. ${ }^{77}$ School of Public Health, Curtin University, Perth, Western Australia, Australia. ${ }^{78} \mathrm{Genomic}$ Medicine Group, Galician Foundation of Genomic Medicine, Instituto de Investigación Sanitaria de Santiago de Compostela (IDIS), Complejo Hospitalario Universitario de Santiago, SERGAS, Santiago de Compostela, Spain. ${ }^{79}$ Moores Cancer Center, University of California San Diego, La Jolla, CA, USA. ${ }^{80}$ Behavioral and Epidemiology Research Group, American Cancer Society, Atlanta, GA, USA. ${ }^{81}$ Medical Oncology Department, Hospital Clínico San Carlos, Instituto de Investigación Sanitaria San Carlos (IdISSC), Centro Investigación Biomédica en Red de Cáncer (CIBERONC), Madrid, Spain. ${ }^{82}$ Cancer Epidemiology Division, Cancer 
Council Victoria, Melbourne, Victoria, Australia. ${ }^{83}$ Centre for Epidemiology and Biostatistics, Melbourne School of Population and Global Health, The University of Melbourne, Melbourne, Victoria, Australia. ${ }^{84}$ Precision Medicine, School of Clinical Sciences at Monash Health, Monash University, Clayton, Victoria, Australia. ${ }^{85}$ Department of Medicine, McGill University, Montréal, OC, Canada. ${ }^{86}$ Division of Clinical Epidemiology, Royal Victoria Hospital, McGill University, Montréal, QC, Canada. ${ }^{87}$ Department of Dermatology, Huntsman Cancer Institute, University of Utah School of Medicine, Salt Lake City, UT, USA. ${ }^{8}$ Cancer \& Environment Group, Center for Research in Epidemiology and Population Health (CESP), INSERM, University Paris-Sud, University Paris-Saclay, Villejuif, France. ${ }^{89}$ Department of Preventive Medicine, Keck School of Medicine, University of Southern California, Los Angeles, CA, USA. ${ }^{90}$ Institute of Environmental Medicine, Karolinska Institutet, Stockholm, Sweden. ${ }^{91}$ Department of Oncology, Södersjukhuset, Stockholm, Sweden. ${ }^{92}$ Centre for Cancer Genetic Epidemiology, Department of Oncology, University of Cambridge, Cambridge, UK. ${ }^{93}$ Department of Health Sciences Research, Mayo Clinic, Rochester, MN, USA. ${ }^{94}$ Saw Swee Hock School of Public Health, National University of Singapore and National University Health System, Singapore, Singapore. ${ }^{95}$ Department of Surgery, National University Health System, Singapore, Singapore. ${ }^{96}$ Department of Surgery, Kaohsiung Municipal Hsiao-Kang Hospital, Kaohsiung, Taiwan. ${ }^{97}$ Program in Genetic Epidemiology and Statistical Genetics, Harvard T.H. Chan School of Public Health, Boston, MA, USA. ${ }^{98}$ Nuffield Department of Population Health, University of Oxford, Oxford, UK. ${ }^{99}$ Center for Clinical Cancer Genetics, The University of Chicago, Chicago, IL, USA. ${ }^{100}$ Division of Cancer Epidemiology and Prevention, Aichi Cancer Center Research Institute, Nagoya, Japan. ${ }^{101}$ Division of Cancer Epidemiology, Nagoya University Graduate School of Medicine, Nagoya, Japan. ${ }^{102}$ Division of Epidemiology, Center for Public Health Sciences, National Cancer Center, Tokyo, Japan. ${ }^{103}$ Research Centre for Genetic Engineering and Biotechnology 'Georgi D. Efremov', MASA, Skopje, Republic of North Macedonia. ${ }^{104}$ Department of Genetics and Pathology, Pomeranian Medical University, Szczecin, Poland. ${ }^{105}$ Independent Laboratory of Molecular Biology and Genetic Diagnostics, Pomeranian Medical University, Szczecin, Poland. ${ }^{106}$ Department of Medicine, Division of Oncology, Stanford Cancer Institute, Stanford University School of Medicine, Stanford, CA, USA. ${ }^{107}$ Department of Preventive Medicine, Seoul National University College of Medicine, Seoul, Korea. ${ }^{108}$ Division of Molecular Pathology, The Netherlands Cancer Institute - Antoni van Leeuwenhoek Hospital, Amsterdam, The Netherlands. ${ }^{109}$ Saint Petersburg State University, Saint-Petersburg, Russia. ${ }^{110}$ Department of Surgery, Daerim Saint Mary's Hospital, Seoul, Korea. ${ }^{111}$ Department of Medical Genetics, Oslo University Hospital and University of Oslo, Oslo, Norway. ${ }^{112}$ Department of Health Research and Policy, Stanford University School of Medicine, Stanford, CA, USA. ${ }^{113}$ Epidemiology Program, University of Hawaii Cancer Center, Honolulu, HI, USA. ${ }^{114}$ Human Genetics Division, Genome Institute of Singapore, Singapore, Singapore. ${ }^{115}$ Department of Molecular Medicine and Surgery, Karolinska Institutet, Stockholm, Sweden. ${ }^{116}$ Department of Clinical Genetics, Karolinska University Hospital, Stockholm, Sweden. ${ }^{117}$ Division of Population Health, Health Services Research and Primary Care, School of Health Sciences, Faculty of Biology, Medicine and Health, The University of Manchester, Manchester, UK. ${ }^{118} \mathrm{Clinical}$ Gerontology, Department of Public Health and Primary Care, University of Cambridge, Cambridge, UK. ${ }^{119}$ Institute of Clinical Medicine, Pathology and Forensic Medicine, University of Eastern Finland, Kuopio, Finland. ${ }^{120}$ Imaging Center, Department of Clinical Pathology, Kuopio University Hospital, Kuopio, Finland. ${ }^{121}$ Molecular Genetics of Breast Cancer, German Cancer Research Center (DKFZ), Heidelberg, Germany. ${ }^{122}$ Unit of Medical Genetics, Department of Medical Oncology and Hematology, Fondazione IRCCS Istituto Nazionale dei Tumori di Milano, Milan, Italy. ${ }^{123}$ Department of Clinical Science and Education, Södersjukhuset, Karolinska Institutet, Stockholm, Sweden. ${ }^{124}$ Cancer Research Malaysia, Subang Jaya, Selangor, Malaysia. ${ }^{125}$ Department of Medical Oncology, University Hospital of Heraklion, Heraklion, Greece. ${ }^{126}$ Department of Gynecology and Obstetrics, University of Munich, Campus Großhadern, Munich, Germany. ${ }^{127} \mathrm{MRC}$ Clinical Trials Unit at UCL, Institute of Clinical Trials \& Methodology, University College London, London, UK. ${ }^{128}$ Department of Laboratory Medicine and Pathobiology, University of Toronto, Toronto, ON, Canada. ${ }^{129}$ Laboratory Medicine Program, University Health Network, Toronto, ON, Canada. ${ }^{130}$ Department of Obstetrics and Gynecology, Helsinki University Hospital, University of Helsinki, Helsinki, Finland. ${ }^{131}$ Clinical Genetics Research Lab, Department of Cancer Biology and Genetics, Memorial Sloan Kettering Cancer Center, New York, NY, USA. ${ }^{132}$ Clinical Genetics Service, Department of Medicine, Memorial Sloan Kettering Cancer Center, New York, NY, USA. ${ }^{133}$ The Breast Cancer Now Toby Robins Research Centre, The Institute of Cancer Research, London, UK. ${ }^{134}$ Centre for Cancer Research and Cell Biology, Queen's University Belfast, Belfast, Ireland, UK. ${ }^{135}$ Genome Diagnostics Program, IFOM - the FIRC Institute for Molecular Oncology, Milan, Italy. ${ }^{136}$ Department of Gynaecology and Obstetrics, University Hospital Ulm, Ulm, Germany. ${ }^{137}$ Clalit National Cancer Control Center, Carmel Medical Center and Technion Faculty of Medicine, Haifa, Israel. ${ }^{138}$ Medical Oncology Department, Hospital Universitario Puerta de Hierro, Madrid, Spain. ${ }^{139}$ Department of Gynaecology and Obstetrics, University Hospital Erlangen, Friedrich-Alexander University Erlangen-Nuremberg, Comprehensive Cancer Center Erlangen-EMN, Erlangen, Germany. ${ }^{140}$ Department of Oncology, University Hospital of Larissa, Larissa, Greece. ${ }^{141}$ Center for Familial Breast and Ovarian Cancer, Faculty of Medicine and University Hospital Cologne, University of Cologne, Cologne, Germany. ${ }^{142}$ Center for Integrated Oncology (CIO), Faculty of Medicine and University Hospital Cologne, University of Cologne, Cologne, Germany. ${ }^{143}$ Center for Molecular Medicine Cologne (CMMC), Faculty of Medicine and University Hospital Cologne, University of Cologne, Cologne, Germany. ${ }^{144}$ National Center for Tumor Diseases, University Hospital and German Cancer Research Center, Heidelberg, Germany. ${ }^{145}$ Institute of Biomedical Sciences, Academia Sinica, Taipei, Taiwan. ${ }^{146}$ School of Public Health, China Medical University, Taichung, Taiwan. ${ }^{147}$ Genomics Center, Centre Hospitalier Universitaire de Québec - Université Laval Research Center, Québec City, OC, Canada. ${ }^{148}$ Department of Clinical Pathology, The University of Melbourne, Melbourne, Victoria, Australia. ${ }^{149}$ Population Oncology, BC Cancer, Vancouver, BC, Canada. ${ }^{150}$ School of Population and Public Health, University of British Columbia, Vancouver, BC, Canada. ${ }^{151}$ Breast Cancer Research Programme, Cancer Research Malaysia, Subang Jaya, Selangor, Malaysia. ${ }^{152}$ Department of Surgery, Faculty of Medicine, University of Malaya, Kuala Lumpur, Malaysia. ${ }^{153}$ Department of Epidemiology, Mailman School of Public Health, Columbia University, New York, NY, USA. ${ }^{154}$ Department of Gynecology and Obstetrics, Helios Clinics Berlin-Buch, Berlin, Germany. ${ }^{155}$ Department of Health Science Research, 
Division of Epidemiology, Mayo Clinic, Rochester, MN, USA. ${ }^{156}$ Department of Clinical Genetics, Leiden University Medical Center, Leiden, The Netherlands. ${ }^{157}$ Department of Surgical Sciences, Uppsala University, Uppsala, Sweden. ${ }^{158}$ Department of Medicine, Institute for Human Genetics, UCSF Helen Diller Family Comprehensive Cancer Center, University of California San Francisco, San Francisco, CA, USA. ${ }^{159}$ Center for Population Health Research, National Institute of Public Health, Cuernavaca, Morelos, Mexico. ${ }^{160}$ Division of Genetics and Epidemiology, The Institute of Cancer Research, London, UK. ${ }^{161}$ Division of Breast Cancer Research, The Institute of Cancer Research, London, UK. ${ }^{162}$ Division of Psychosocial Research and Epidemiology, The Netherlands Cancer Institute - Antoni van Leeuwenhoek hospital, Amsterdam, The Netherlands. *Lists of authors and their affiliations appear at the end of the paper. 凶e-mail: a.hollestelle@erasmusmc.nl

In breast cancer, high levels of homeobox protein Hox-B13 (HOXB13) have been associated with disease progression of ER-positive breast cancer patients and resistance to tamoxifen treatment. Since HOXB13 p.G84E is a prostate cancer risk allele, we evaluated the association between HOXB13 germline mutations and breast cancer risk in a previous study consisting of 3,270 familial non-BRCA1/2 breast cancer cases and 2,327 controls from the Netherlands. Although both recurrent $H O X B 13$ mutations p.G84E and p.R217C were not associated with breast cancer risk, the risk estimation for p.R217C was not very precise. To provide more conclusive evidence regarding the role of HOXB13 in breast cancer susceptibility, we here evaluated the association between $H O X B 13$ mutations and increased breast cancer risk within 81 studies of the international Breast Cancer Association Consortium containing 68,521 invasive breast cancer patients and 54,865 controls. Both HOXB13 p.G84E and p.R217C did not associate with the development of breast cancer in European women, neither in the overall analysis $(\mathrm{OR}=1.035,95 \% \mathrm{Cl}=0.859-1.246, P=0.718$ and $\mathrm{OR}=0.798,95 \% \mathrm{Cl}=0.482-1.322, P=0.381$ respectively), nor in specific high-risk subgroups or breast cancer subtypes. Thus, although involved in breast cancer progression, $H O X B 13$ is not a material breast cancer susceptibility gene.

Breast cancer is a complex disease and several classes of germline variants have been identified that together explain about half of the total genetic heritability of breast cancer. These include rare germline mutations in high and moderate penetrance breast cancer susceptibility genes BRCA1, BRCA2, CDH1, PTEN, STK11, TP53, $P A L B 2, A T M, C H E K 2$ and $N B N^{1}$. In addition, genome-wide association studies (GWASs) have identified over 170 common low penetrance alleles each conferring a small increased risk to develop breast cancer ${ }^{2,3}$. Importantly, the risks these low penetrance alleles confer combine multiplicatively and since these variants are so common in the population women in the top $1 \%$ of risk have a 4.4 - and 2.8-fold increased risk to develop ER-positive and ER-negative breast cancer, respectively ${ }^{4}$. Still, to identify better those women at risk for developing breast cancer and establish more precise risk estimates, we need to explain the remainder of the genetic heritability of breast cancer.

In this respect, the rare HOXB13 p.G84E germline mutation (i.e. NM_006361.6:c.251 G > A; NP_006352.2:p. (G84E); rs 138213197:C > T) was found to be associated with an increased risk to develop prostate cancer by linkage analysis and candidate gene sequencing of 200 genes at the 17q21-22 linkage region ${ }^{5}$. Since then, several studies have validated this association and meta-analyses have shown the prostate cancer risk to be 3- to 4-fold increased for male carriers ${ }^{6-8}$. Moreover, the p.G84E mutation also associated with early-onset prostate cancer, multiple affected relatives and highly aggressive disease ${ }^{6,8}$. Considering the evidence, there is a strong consensus for including the $H O X B 13$ gene in genetic testing for hereditary prostate cancer'.

In recent years, we have also begun to understand the role of HOXB13 in prostate cancer progression. HOXB13 acts as a transcription factor and, together with the androgen receptor (AR) and FOXA1, regulates expression of the RFX6 gene which encodes a driver of prostate cancer progression. Interestingly, HOXB13 is preferentially recruited to the risk allele of a prostate cancer risk associated SNP, rs339331, located in an enhancer element upstream of RFX6, thereby enhancing RFX6 expression and promoting more aggressive disease ${ }^{10}$. Moreover, HOXB13 also pioneers binding of the constitutively active splice variant 7 of the androgen receptor (AR-V7) to open chromatin of castrate-resistant prostate cancer (CRPC) genomes to upregulate target oncogenes $^{11}$. Importantly, AR-V7 plays an important role in the anti-AR therapy resistance ${ }^{12}$.

In breast cancer, HOXB13 also plays an important role in disease progression. A high HOXB13 to IL17BR expression ratio was associated with a high risk of recurrence and poor outcome for estrogen receptor (ER)-positive breast cancer patients ${ }^{13-15}$. Furthermore, high expression of HOXB13 predicted a poor response to tamoxifen therapy by suppressing ER and activating the mTOR pathway via IL6 ${ }^{16,17}$. Interestingly, a significant fraction of breast cancer risk SNPs have been found to alter the affinity of chromatin for pioneer factor FOXA1 with which HOXB13 interacts in prostate cancer cells ${ }^{10,18}$. To date, several studies have investigated the association between the germline HOXB13 p.G84E mutation and breast cancer risk, however, these have led to contradictory results ${ }^{7,19-21}$.

In a previous study, we have sequenced the entire coding region of $H O X B 13$ in 1,250 familial non-BRCA1/2 breast cancer cases and 800 controls. We identified two recurrent HOXB13 mutations in the female Dutch population, the known prostate cancer risk allele p.G84E, but also p.R217C (i.e. NM_006361.6:c.649C > T; NP_006352.2:p.(R217C); rs139475791:G > A). We found that neither p.G84E nor p.R217C were associated with an increased breast cancer risk $(\mathrm{OR}=0.81,95 \% \mathrm{CI}=0.41-1.59, P=0.54$ and $\mathrm{OR}=3.57,95 \% \mathrm{CI}=0.76-33.57$, $P=0.14$, respectively) in 3,270 familial non-BRCA1/2 breast cancer patients and 2,327 controls ${ }^{22}$. Considering the low carrier allele frequency (CAF; $0.09 \%$ in controls) and the very wide confidence intervals for the association between $\mathrm{p} . \mathrm{R} 217 \mathrm{C}$ and breast cancer risk, larger studies are needed to provide more conclusive evidence. 


\begin{tabular}{|c|c|c|c|c|c|c|}
\hline & N Controls & N Cases & \begin{tabular}{|l|} 
CAF (\%) \\
Controls
\end{tabular} & \begin{tabular}{|l|} 
CAF (\%) \\
Cases
\end{tabular} & OR $(95 \% \mathrm{CI})^{*}$ & $P$-value* \\
\hline \multicolumn{7}{|l|}{ Overall analysis } \\
\hline Europeans & 44,298 & 54,731 & 0.510 & 0.471 & $1.03(0.86-1.24)$ & 0.74 \\
\hline \multicolumn{7}{|l|}{ Subgroup analysis } \\
\hline \multicolumn{7}{|l|}{ Age of diagnosis } \\
\hline$<50$ years & 44,298 & 17,641 & 0.510 & 0.431 & $0.99(0.72-1.35)$ & 0.98 \\
\hline \multicolumn{7}{|l|}{ Menopausal status } \\
\hline Premenopausal & 44,298 & 12,134 & 0.510 & 0.503 & $1.20(0.88-1.64)$ & 0.24 \\
\hline \multicolumn{7}{|l|}{ Family history } \\
\hline $1^{\text {st }}$ degree relative with $\mathrm{BC}$ & 41,876 & 7,582 & 0.533 & 0.462 & $1.04(0.72-1.51)$ & 0.83 \\
\hline \multicolumn{7}{|l|}{ Second BC } \\
\hline Contralateral BC & 38,310 & 2,144 & 0.506 & 0.373 & $1.00(0.48-2.10)$ & 0.99 \\
\hline \multicolumn{7}{|l|}{ Receptor status } \\
\hline ER positive & 44,298 & 35,969 & 0.510 & 0.442 & $0.98(0.80-1.21)$ & 0.88 \\
\hline ER negative & 44,298 & 9,343 & 0.510 & 0.503 & $1.21(0.87-1.68)$ & 0.26 \\
\hline Triple negative & 44,298 & 4,017 & 0.510 & 0.448 & $1.26(0.76-2.06)$ & 0.37 \\
\hline
\end{tabular}

Table 1. Association of HOXB13 p.G84E with breast cancer risk in women of European descent. N, number; $\mathrm{CAF}$, carrier allele frequency; $\mathrm{OR}$, odds ratio; $\mathrm{CI}$, confidence interval; $\mathrm{BC}$, breast cancer; $\mathrm{ER}$, estrogen receptor. *Dominant genetic model adjusted for country, age and principal components. Not all BCAC studies had info on all variables.

Furthermore, we wanted to replicate our findings for the p.G84E mutation. Therefore, we have genotyped 68,521 breast cancer cases and 54,865 controls from 81 studies in the Breast Cancer Association Consortium (BCAC) for the HOXB13 p.G84E and p.R217C mutations.

\section{Results}

The CAF for the p.G84E mutation varies among different populations. In Asian and African BCAC studies, the p.G84E mutation was not detected, while the CAF was highest in Northern European countries (i.e. Sweden, Denmark and the Netherlands in controls) (Supplementary Table S1). Therefore, we restricted our analysis for the p.G84E mutation to 54,731 cases and 44,298 controls from European countries with a CAF that was larger than zero. In the overall analysis, the p.G84E mutation was not associated with breast cancer risk in Europeans $(\mathrm{OR}=1.033,95 \% \mathrm{CI}=0.857-1.244, P=0.734$; Table 1$)$ in agreement with our previous study. We also performed analyses in which we enriched for high-risk subgroups such as women who were diagnosed before 50 years of age, premenopausal women and women with a family history of breast cancer or contralateral breast cancer. We also performed analyses by receptor status to evaluate whether HOXB13 p.G84E associates with subtype-specific breast cancer risk. However, we did neither find any association between HOXB13 p.G84E and the risk of breast cancer in any of these high-risk subgroups, nor did we find an association with subtype-specific breast cancer risk (Table 1).

Although in our previous study we found that the HOXB13 p.R217C mutation was 3.5 -fold more prevalent in cases than controls, the association between p.R217C and breast cancer risk was not statistically significant and the estimation of the risk was not very precise. Therefore, we evaluated the association of p.R217C with breast cancer risk in the 81 BCAC studies. Similar to p.G84E, the CAF for p.R217C varied among different populations. It was absent in both cases and controls of Asian ancestry, but not those of European and African ancestry. The CAF was highest in Macedonia, the Netherlands and Greece in controls (Supplementary Table S1). We analyzed 54,752 breast cancer patients and 44,422 controls from European countries with a CAF that was larger than zero. In the overall analysis, p.R217C was not associated with an increased breast cancer risk in European women $(\mathrm{OR}=0.798,95 \% \mathrm{CI}=0.482-1.322, P=0.381$; Table 2$)$. Likewise, high-risk subgroup analyses and analyses by receptor status also did not reveal any association between HOXB13 p.R217C and (subtype-specific) breast cancer risk (Table 2).

In our previous study we had sequenced the entire coding region of $H O X B 13$ in 1,250 familial non-BRCA1/2 breast cancer patients and 800 controls and identified two other, less frequent, HOXB13 missense mutations: p.P190L (i.e. NM_006361.6: c.569 C > T; NP_006352.2:p.(P190L)) and p.R268Q (i.e. NM_006361.6:c.803 G > A; NP_006352.2:p.(R268Q); rs748782183:C $>\bar{T})^{22}$. These two mutations had not been investigated before due to their low frequency in the Dutch population. However, the present study enabled us to assess their frequency in a global context. The p.P190L mutation was most prevalent in the African population and absent in the Asian population (Supplementary Table S1). In the Europeans, we identified only four breast cancer patients and four controls carrying this mutation. The low population frequency in Europeans and the low sample size in Africans precluded any reliable analysis of an association with breast cancer risk. The p.R268Q mutation was absent in Asian and African BCAC studies. In Europeans, we identified only two breast cancer patients and two controls carrying this mutation, again precluding any reliable analysis of an association with breast cancer risk (Supplementary Table S1). 


\begin{tabular}{|c|c|c|c|c|c|c|}
\hline & N Controls & N Cases & $\begin{array}{l}\text { CAF (\%) } \\
\text { Controls }\end{array}$ & $\begin{array}{l}\text { CAF (\%) } \\
\text { Cases }\end{array}$ & OR $(95 \% \mathrm{CI}) *$ & $P$-value* \\
\hline \multicolumn{7}{|l|}{ Overall analysis } \\
\hline Europeans & 44,422 & 54,752 & 0.077 & 0.062 & $0.80(0.48-1.32)$ & 0.38 \\
\hline \multicolumn{7}{|l|}{ Subgroup analysis } \\
\hline \multicolumn{7}{|l|}{ Age of diagnosis } \\
\hline$<50$ years & 44,422 & 17,669 & 0.077 & 0.045 & $0.38(0.14-1.01)$ & 0.05 \\
\hline \multicolumn{7}{|l|}{ Menopausal status } \\
\hline Premenopausal & 44,422 & 12,195 & 0.077 & 0.057 & $0.59(0.24-1.44)$ & 0.25 \\
\hline \multicolumn{7}{|l|}{ Family history } \\
\hline $1^{\text {st }}$ degree relative with $\mathrm{BC}$ & 41,909 & 7,531 & 0.069 & 0.013 & $0.21(0.03-1.53)$ & 0.12 \\
\hline \multicolumn{7}{|l|}{ Second BC } \\
\hline Contralateral BC & 38,346 & 2,137 & 0.076 & 0.047 & $0.43(0.05-3.43)$ & 0.43 \\
\hline \multicolumn{7}{|l|}{ Receptor status } \\
\hline ER positive & 44,422 & 35,930 & 0.077 & 0.061 & $0.81(0.46-1.42)$ & 0.46 \\
\hline ER negative & 44,422 & 9,343 & 0.077 & 0.064 & $0.82(0.33-2.03)$ & 0.66 \\
\hline Triple negative & 44,422 & 4,045 & 0.077 & 0.025 & $0.29(0.04-2.19)$ & 0.23 \\
\hline
\end{tabular}

Table 2. Association of $H O X B 13$ p.R217C with breast cancer risk in women of European descent. N, number; $\mathrm{CAF}$, carrier allele frequency; OR, odds ratio; $\mathrm{CI}$, confidence interval; $\mathrm{BC}$, breast cancer; $\mathrm{ER}$, estrogen receptor. *Dominant genetic model adjusted for country, age and principal components. Not all BCAC studies had info on all variables.

\section{Discussion}

We genotyped four HOXB13 missense mutations: p.G84E, p.P190L, p.R217C and p.R268Q in 68,521 breast cancer cases and 54,865 controls from 81 studies in the BCAC on the OncoArray. All mutations were present in Europeans, but not in Asians. The p.P190L and p.R217C mutations were also present in the African ancestry BCAC studies, but not p.G84E and p.R268Q. Both p.P190L and p.R268Q were too rare to be evaluated for their association with an increased breast cancer risk. There were sufficient carriers of HOXB13 p.G84E and p.R217C to allow association analysis in Europeans, however, both mutations did not associate with breast cancer risk. Our study, by contrast with prostate cancer, shows that $H O X B 13$ is not a material breast cancer susceptibility gene.

The current study is by far the largest study that has been performed evaluating the association with an increased breast cancer risk for germline $H O X B 13$ mutation carriers. Previously, Alanee et al. had found evidence that $H O X B 13$ p.G84E conferred an increased breast cancer risk in 877 familial non-BRCA1/2 mutation carriers and 1650 controls $(\mathrm{OR}=5.7,95 \% \mathrm{CI}=1.0-40.7, P=0.02)^{19}$. However, in a larger study conducted by Akbari et al., no such association between the p.G84E mutation and an increased breast cancer risk was observed among 4,037 cases, of which 1,082 were familial, and 2,762 controls $(\mathrm{OR}=1.2,95 \% \mathrm{CI}=0.34-4.1, P=1.0)^{20}$. A study by Laitinen et al. consisting of 986 breast cancer patients (i.e. 323 familial non-BRCA1/2 carriers and 663 unselected breast cancer patients) and 1,449 controls also did not reveal an association for overall breast cancer risk and p.G84E among Finnish women ${ }^{21}$. Results of these three studies have been pooled in a fixed-effects meta-analysis by Cai et al. and did not find a significant association between HOXB13 p.G84E and an increased breast cancer risk $(\mathrm{OR}=1.42,95 \% \mathrm{CI}=0.78-2.61, P=0.26)^{7}$. We also did not observe an increased breast cancer risk associated with the p.G84E mutation in our previous study of 3,270 familial non-BRCA1/2 breast cancer cases and 2,327 controls $(\mathrm{OR}=0.81,95 \% \mathrm{CI}=0.41-1.59, P=0.54)^{22}$. The results of the current study concur with these observations in that HOXB13 p.G84E does not appear to act as a breast cancer susceptibility allele, neither in overall analyses $(\mathrm{OR}=1.035,95 \% \mathrm{CI}=0.859-1.246, P=0.718)$ nor in analyses enriching for particular (high-risk) subgroups.

Besides p.G84E, we also identified p.R217C to be a recurrent mutation in the female Dutch population ${ }^{22}$. Since the estimation of the breast cancer risk for this mutation was not very precise in our previous study, we sought to re-evaluate the association between p.R217C and increased breast cancer risk in the current study. As for p.G84E, we did not find any association between p.R217C and an increased breast cancer risk, neither in overall analyses $(\mathrm{OR}=0.798,95 \% \mathrm{CI}=0.482-1.322, P=0.381)$, nor in subgroup analyses. Interestingly, the p.R217C mutation had been described before among a few prostate cancer cases, but Xu et al. reported that p.R217C did not co-segregate with prostate cancer in the two families they identified ${ }^{23,24}$. In concordance with this, OncoArray summary association results from the PRACTICAL consortium show that, indeed, p.R217C is also not a material prostate cancer susceptibility allele $(\mathrm{OR}=1.32,95 \% \mathrm{CI}=0.57-2.07)$, while p.G84E is associated with an increased prostate cancer risk in this data set $(\mathrm{OR}=4.23,95 \% \mathrm{CI}=4.03-4.42)^{25}$.

Although HOXB13 plays an important role in both breast and prostate cancer progression ${ }^{10,11,13-17}$, germline mutations in the HOXB13 gene seem to associate with the development of prostate cancer only ${ }^{5-8}$. This suggests distinct biological pathways associated with HOXB13 function in breast and prostate tissue. In prostate cancer, HOXB13 co-localizes with AR and acts as a repressor of AR target genes to modulate AR hormonal responses ${ }^{26,27}$. In breast cancer, ER and HOXB13 have been shown to regulate each other's expression ${ }^{17,28}$. Thus, in both tissue types hormonal responses are closely interlinked with HOXB13 function. More research is needed, however, to understand better the differential roles of HOXB13 in disease initiation and progression. 
To conclude, in our large study consisting of 68,521 invasive breast cancer cases and 54,865 controls from 81 BCAC studies we provide strong evidence that the rare, but recurrent HOXB13 germline mutations p.G84E and p.R217C are not associated with an increased risk to develop breast cancer. HOXB13 is therefore not a material breast cancer susceptibility gene.

\section{Materials and Methods}

Study population. In this study, BCAC consists of 81 case-control studies of unrelated women with participants of European, Asian and African ancestry contributing 68,521 patients with invasive breast cancer and 54,865 controls $^{2,3}$. All studies provided core data on disease status and age at diagnosis while only a subset of the studies provided data on menopausal status, ER, PR and ERBB2 status, family history and bilateral breast cancer. All 81 BCAC studies were approved by their relevant governing research ethics committee and all participants provided written informed consent. The experimental protocol was approved by the Medical Ethical Committee of the Erasmus Medical Center Rotterdam and the study was carried out in accordance with the Code of Conduct of the Federation of Medical Scientific Societies in the Netherlands (https://www.federa.org/gedragscodes).

OncoArray genotyping. Genotyping of the 81 BCAC studies was performed previously using the OncoArray, a custom-designed Illumina Infinium BeadChip. About half of the approximately 533,000 OncoArray SNPs were selected as a 'GWAS backbone' (Illumina HumanCore) with the remainder of SNPs selected by the disease-based consortia representing the main cancer sites (e.g. breast, ovarian, prostate, lung, colorectal) for several distinct reasons as detailed $\mathrm{in}^{29}$. Approximately 72,000 SNPs were selected specifically for their relevance to breast cancer. Details of the genotype calling and quality control for OncoArray are described elsewhere ${ }^{2,29}$. In brief, samples were excluded when the call rate was below $95 \%$ or when these were probable duplicates, close relatives or samples with extreme heterozygosity. Ancestry was computed using a principal component analysis (PCA). Variants were excluded using the following criteria: an overall call rate $<99 \%$ or $<95 \%$ in any consortium, minor allele frequency $(\mathrm{MAF})<0.001$, poor intensity and clustering metrics, deviation from the expected frequency as observed in the 1000 Genomes Project and deviation from the Hardy-Weinberg equilibrium (HWE; $P<10^{-7}$ in controls or $P<10^{-12}$ in cases). A total of 494,763 SNPs passed the quality control and included the following four HOXB13 missense variants: c.251 G > A (p.G84E; rs138213197), c.569 C > T (p.P190L), c.649 C > T (p.R217C; rs139475791) and c.803 G > A (p.R268Q).

Statistical analyses. The association between $H O X B 13$ mutations and invasive breast cancer risk was evaluated using dominant genetic models by logistic regression analysis adjusting for country, age and principal components in European women. Subgroup analyses for the p.G84E and p.R217C variants were based on enriching for high-risk subgroups (i.e women diagnosed with breast cancer $<50$ years, premenopausal women, women with a family history of breast cancer (i.e. $1^{\text {st }}$ degree relative with breast cancer) and women diagnosed with a contralateral breast cancer) as well as stratification for hormone receptor status (i.e. ER positive, ER negative, triple negative) to evaluate subtype-specific breast cancer risk. All $P$-values were two-sided and $P<0.05$ was considered to be statistically significant after correction for multiple testing by the Bonferroni procedure. Logistic regression analyses were performed using $\mathrm{R}$ version 3.3.3.

\section{Data availability}

OncoArray summary statistics from the BCAC are available at http://bcac.ccge.medschl.cam.ac.uk/bcacdata/ oncoarray/gwas-icogs-and-oncoarray-summary-results/. Per-sample genotype data, core demographic data and data on diagnosis and pathology can be requested via the BCAC Data Access Co-ordinating Committee (DACC) at http://bcac.ccge.medschl.cam.ac.uk/bcacdata/. OncoArray summary statistics from the PRACTICAL consortium are available at http://practical.icr.ac.uk/blog/?page_id=8088.

Received: 24 January 2020; Accepted: 22 April 2020;

Published online: 16 June 2020

\section{References}

1. Easton, D. F. et al. Gene-panel sequencing and the prediction of breast-cancer risk. N Engl J Med 372, 2243-2257, https://doi. org/10.1056/NEJMsr1501341 (2015).

2. Michailidou, K. et al. Association analysis identifies 65 new breast cancer risk loci. Nature 551, 92-94, https://doi.org/10.1038/ nature24284 (2017).

3. Milne, R. L. et al. Identification of ten variants associated with risk of estrogen-receptor-negative breast cancer. Nat Genet 49, 1767-1778, https://doi.org/10.1038/ng.3785 (2017).

4. Mavaddat, N. et al. Polygenic Risk Scores for Prediction of Breast Cancer and Breast Cancer Subtypes. Am J Hum Genet 104, 21-34, https://doi.org/10.1016/j.ajhg.2018.11.002 (2019).

5. Ewing, C. M. et al. Germline mutations in HOXB13 and prostate-cancer risk. $N$ Engl J Med 366, 141-149, https://doi.org/10.1056/ NEJMoa1110000 (2012).

6. Huang, H. \& Cai, B. G84E mutation in HOXB13 is firmly associated with prostate cancer risk: a meta-analysis. Tumour Biol 35, 1177-1182, https://doi.org/10.1007/s13277-013-1157-5 (2014).

7. Cai, Q. et al. Germline HOXB13 p.Gly84Glu mutation and cancer susceptibility: a pooled analysis of 25 epidemiological studies with 145,257 participates. Oncotarget 6, 42312-42321, https://doi.org/10.18632/oncotarget.5994 (2015).

8. Zhang, J. et al. Association between germline homeobox B13 (HOXB13) G84E allele and prostate cancer susceptibility: a metaanalysis and trial sequential analysis. Oncotarget 7, 67101-67110, https://doi.org/10.18632/oncotarget.11937 (2016).

9. Giri, V. N. et al. Role of Genetic Testing for Inherited Prostate Cancer Risk: Philadelphia Prostate Cancer Consensus Conference 2017. J Clin Oncol 36, 414-424, https://doi.org/10.1200/JCO.2017.74.1173 (2018).

10. Huang, Q. et al. A prostate cancer susceptibility allele at $6 \mathrm{q} 22$ increases RFX6 expression by modulating HOXB13 chromatin binding. Nat Genet 46, 126-135, https://doi.org/10.1038/ng.2862 (2014). 
11. Chen, Z. et al. Diverse AR-V7 cistromes in castration-resistant prostate cancer are governed by HoxB13. Proc Natl Acad Sci U S A 115, 6810-6815, https://doi.org/10.1073/pnas.1718811115 (2018).

12. Maughan, B. L. \& Antonarakis, E. S. Clinical Relevance of Androgen Receptor Splice Variants in Castration-Resistant Prostate Cancer. Curr Treat Options Oncol 16, 57, https://doi.org/10.1007/s11864-015-0375-z (2015).

13. Ma, X. J. et al. A two-gene expression ratio predicts clinical outcome in breast cancer patients treated with tamoxifen. Cancer Cell 5 , 607-616, https://doi.org/10.1016/j.ccr.2004.05.015 (2004).

14. Jansen, M. P. et al. HOXB13-to-IL17BR expression ratio is related with tumor aggressiveness and response to tamoxifen of recurrent breast cancer: a retrospective study. J Clin Oncol 25, 662-668, https://doi.org/10.1200/JCO.2006.07.3676 (2007).

15. Zhao, L., Zhu, S., Gao, Y. \& Wang, Y. Two-gene expression ratio as predictor for breast cancer treated with tamoxifen: evidence from meta-analysis. Tumour Biol 35, 3113-3117, https://doi.org/10.1007/s13277-013-1403-x (2014).

16. Jerevall, P. L. et al. Predictive relevance of HOXB13 protein expression for tamoxifen benefit in breast cancer. Breast Cancer Res 12, R53, https://doi.org/10.1186/bcr2612 (2010).

17. Shah, N. et al. HOXB13 mediates tamoxifen resistance and invasiveness in human breast cancer by suppressing ERalpha and inducing IL-6 expression. Cancer Res 73, 5449-5458, https://doi.org/10.1158/0008-5472.CAN-13-1178 (2013).

18. Cowper-Sal lari, R. et al. Breast cancer risk-associated SNPs modulate the affinity of chromatin for FOXA1 and alter gene expression. Nat Genet 44, 1191-1198, https://doi.org/10.1038/ng.2416 (2012).

19. Alanee, S., Couch, F. \& Offit, K. Association of a HOXB13 variant with breast cancer. N Engl J Med 367, 480-481, https://doi. org/10.1056/NEJMc1205138 (2012).

20. Akbari, M. R. et al. The HOXB13 p.Gly84Glu mutation is not associated with the risk of breast cancer. Breast Cancer Res Treat 136, 907-909, https://doi.org/10.1007/s10549-012-2295-y (2012).

21. Laitinen, V. H. et al. HOXB13 G84E mutation in Finland: population-based analysis of prostate, breast, and colorectal cancer risk. Cancer Epidemiol Biomarkers Prev 22, 452-460, https://doi.org/10.1158/1055-9965.EPI-12-1000-T (2013).

22. Liu, J. et al. Recurrent HOXB13 mutations in the Dutch population do not associate with increased breast cancer risk. Sci Rep 6, 30026, https://doi.org/10.1038/srep30026 (2016).

23. Xu, J. et al. HOXB13 is a susceptibility gene for prostate cancer: results from the International Consortium for Prostate Cancer Genetics (ICPCG). Hum Genet 132, 5-14, https://doi.org/10.1007/s00439-012-1229-4 (2013).

24. Karlsson, R. et al. A population-based assessment of germline HOXB13 G84E mutation and prostate cancer risk. Eur Urol 65, 169-176, https://doi.org/10.1016/j.eururo.2012.07.027 (2014).

25. Schumacher, F. R. et al. Association analyses of more than 140,000 men identify 63 new prostate cancer susceptibility loci. Nat Genet 50, 928-936, https://doi.org/10.1038/s41588-018-0142-8 (2018).

26. Norris, J. D. et al. The homeodomain protein HOXB13 regulates the cellular response to androgens. Mol Cell 36, 405-416, https:// doi.org/10.1016/j.molcel.2009.10.020 (2009).

27. Kim, S. D. et al. HOXB13 is co-localized with androgen receptor to suppress androgen-stimulated prostate-specific antigen expression. Anat Cell Biol 43, 284-293, https://doi.org/10.5115/acb.2010.43.4.284 (2010).

28. Wang, Z. et al. The prognostic biomarkers HOXB13, IL17BR, and CHDH are regulated by estrogen in breast cancer. Clin Cancer Res 13, 6327-6334, https://doi.org/10.1158/1078-0432.CCR-07-0310 (2007).

29. Amos, C. I. et al. The OncoArray Consortium: A Network for Understanding the Genetic Architecture of Common Cancers. Cancer Epidemiol Biomarkers Prev 26, 126-135, https://doi.org/10.1158/1055-9965.EPI-16-0106 (2017).

\section{Acknowledgements}

We thank all the individuals who took part in these studies and all the researchers, clinicians, technicians and administrative staff who have enabled this work to be carried out. ABCFS thank Maggie Angelakos, Judi Maskiell, Gillian Dite. ABCS thanks the Blood bank Sanquin, The Netherlands. ABCTB samples are made available to researchers on a non-exclusive basis. The ACP study wishes to thank the participants in the Thai Breast Cancer study. Special thanks also go to the Thai Ministry of Public Health (MOPH), doctors and nurses who helped with the data collection process. Finally, the study would like to thank Dr. Prat Boonyawongviroj, the former Permanent Secretary of MOPH and Dr. Pornthep Siriwanarungsan, the former Department Director-General of Disease Control who have supported the study throughout. BBCS thanks Eileen Williams, Elaine Ryder-Mills, Kara Sargus. BCEES thanks Allyson Thomson, Christobel Saunders, Terry Slevin, BreastScreen Western Australia, Elizabeth Wylie, Rachel Lloyd. The BCINIS study would not have been possible without the contributions of Dr. K. Landsman, Dr. N. Gronich, Dr. A. Flugelman, Dr. W. Saliba, Dr. E. Liani, Dr. I. Cohen, Dr. S. Kalet, Dr. V. Friedman, Dr. O. Barnet of the NICCC in Haifa, and all the contributing family medicine, surgery, pathology and oncology teams in all medical institutes in Northern Israel. The BREOGAN study would not have been possible without the contributions of the following: Manuela Gago-Dominguez, Jose Esteban Castelao, Angel Carracedo, Victor Muñoz Garzón, Alejandro Novo Domínguez, Maria Elena Martinez, Sara Miranda Ponte, Carmen Redondo Marey, Maite Peña Fernández, Manuel Enguix Castelo, Maria Torres, Manuel Calaza (BREOGAN), José Antúnez, Máximo Fraga and the staff of the Department of Pathology and Biobank of the University Hospital Complex of Santiago-CHUS, Instituto de Investigación Sanitaria de Santiago, IDIS, Xerencia de Xestion Integrada de Santiago-SERGAS; Joaquín González-Carreró and the staff of the Department of Pathology and Biobank of University Hospital Complex of Vigo, Instituto de Investigacion Biomedica Galicia Sur, SERGAS, Vigo, Spain. BSUCH thanks Peter Bugert, Medical Faculty Mannheim. The CAMA study would like to recognize CONACyT for the financial support provided for this work and all physicians responsible for the project in the different participating hospitals: Dr. Germán Castelazo (IMSS, Ciudad de México, DF), Dr. Sinhué Barroso Bravo (IMSS, Ciudad de México, DF), Dr. Fernando Mainero Ratchelous (IMSS, Ciudad de México, DF), Dr. Joaquín Zarco Méndez (ISSSTE, Ciudad de México, DF), Dr. Edelmiro Pérez Rodríguez (Hospital Universitario, Monterrey, Nuevo León), Dr. Jesús Pablo Esparza Cano (IMSS, Monterrey, Nuevo León), Dr. Heriberto Fabela (IMSS, Monterrey, Nuevo León), Dr. Fausto Hernández Morales (ISSSTE, Veracruz, Veracruz), Dr. Pedro Coronel Brizio (CECAN SS, Xalapa, Veracruz) and Dr. Vicente A. Saldaña Quiroz (IMSS, Veracruz, Veracruz). CBCS thanks study participants, co-investigators, collaborators and staff of the Canadian Breast Cancer Study, and project coordinators Agnes Lai and Celine Morissette. CCGP thanks Styliani Apostolaki, Anna Margiolaki, Georgios Nintos, Maria Perraki, Georgia Saloustrou, Georgia Sevastaki, Konstantinos Pompodakis. CGPS thanks staff and participants of the Copenhagen General Population Study. For the excellent technical assistance: Dorthe Uldall Andersen, Maria Birna Arnadottir, Anne Bank, Dorthe Kjeldgård Hansen. The Danish Cancer Biobank is acknowledged for providing infrastructure for the collection of blood samples for the cases. 
COLBCCC thanks all patients, the physicians Justo G. Olaya, Mauricio Tawil, Lilian Torregrosa, Elias Quintero, Sebastian Quintero, Claudia Ramírez, José J. Caicedo, and Jose F. Robledo, the researchers Fabian Gil, Angela Umana, Angela Beltran and Viviana Ariza, and the technician Michael Gilbert for their contributions and commitment to this study. Investigators from the CPS-II cohort thank the participants and Study Management Group for their invaluable contributions to this research. They also acknowledge the contribution to this study from central cancer registries supported through the Centers for Disease Control and Prevention National Program of Cancer Registries, as well as cancer registries supported by the National Cancer Institute Surveillance Epidemiology and End Results program. The CTS Steering Committee includes Leslie Bernstein, Susan Neuhausen, James Lacey, Sophia Wang, Huiyan Ma, and Jessica Clague DeHart at the Beckman Research Institute of City of Hope, Dennis Deapen, Rich Pinder, and Eunjung Lee at the University of Southern California, Pam Horn-Ross, Peggy Reynolds, Christina Clarke Dur and David Nelson at the Cancer Prevention Institute of California, Hoda Anton-Culver, Argyrios Ziogas, and Hannah Park at the University of California Irvine, and Fred Schumacher at Case Western University. DIETCOMPLYF thanks the patients, nurses and clinical staff involved in the study. The DietCompLyf study was funded by the charity Against Breast Cancer (Registered Charity Number 1121258) and the NCRN. We thank the participants and the investigators of EPIC (European Prospective Investigation into Cancer and Nutrition). ESTHER thanks Hartwig Ziegler, Sonja Wolf, Volker Hermann, Christa Stegmaier, Katja Butterbach. GC-HBOC thanks Stefanie Engert, Heide Hellebrand, Sandra Kröber and LIFE - Leipzig Research Centre for Civilization Diseases (Markus Loeffler, Joachim Thiery, Matthias Nüchter, Ronny Baber). The GENICA Network: Dr. Margarete Fischer-Bosch-Institute of Clinical Pharmacology, Stuttgart, and University of Tübingen, Germany [HB, Wing-Yee Lo], German Cancer Consortium (DKTK) and German Cancer Research Center (DKFZ), Partner Site Tübingen, Tübingen, Germany [HB], gefördert durch die Deutsche Forschungsgemeinschaft (DFG) im Rahmen der Exzellenzstrategie des Bundes und der Länder - EXC 2180 - 390900677 [HB], Department of Internal Medicine, Evangelische Kliniken Bonn gGmbH, Johanniter Krankenhaus, Bonn, Germany [Yon-Dschun Ko, Christian Baisch], Institute of Pathology, University of Bonn, Germany [Hans-Peter Fischer], Molecular Genetics of Breast Cancer, Deutsches Krebsforschungszentrum (DKFZ), Heidelberg, Germany [Ute Hamann], Institute for Prevention and Occupational Medicine of the German Social Accident Insurance, Institute of the Ruhr University Bochum (IPA), Bochum, Germany [TB, Beate Pesch, Sylvia Rabstein, Anne Lotz]; and Institute of Occupational Medicine and Maritime Medicine, University Medical Center Hamburg-Eppendorf, Germany [Volker Harth]. HABCS thanks Michael Bremer. HEBCS thanks Sofia Khan, Johanna Kiiski, Carl Blomqvist, Irja Erkkilä. HMBCS thanks Peter Hillemanns, Hans Christiansen and Johann H. Karstens. HUBCS thanks Shamil Gantsev. KARMA thanks the Swedish Medical Research Counsel. KBCP thanks Eija Myöhänen, Helena Kemiläinen. We thank all investigators of the KOHBRA (Korean Hereditary Breast Cancer) Study. LMBC thanks Gilian Peuteman, Thomas Van Brussel, Evy Vanderheyden and Kathleen Corthouts. MABCS thanks Milena Jakimovska (RCGEB "Georgi D. Efremov"), Emilija Lazarova (University Clinic of Radiotherapy and Oncology), Katerina Kubelka-Sabit, Mitko Karadjozov (Adzibadem-Sistina Hospital), Andrej Arsovski and Liljana Stojanovska (Re-Medika Hospital) for their contributions and commitment to this study. MARIE thanks Petra Seibold, Dieter Flesch-Janys, Judith Heinz, Nadia Obi, Alina Vrieling, Sabine Behrens, Ursula Eilber, Muhabbet Celik, Til Olchers and Stefan Nickels. MBCSG (Milan Breast Cancer Study Group): Bernard Peissel, Jacopo Azzollini, Dario Zimbalatti, Daniela Zaffaroni, Irene Feroce, Mariarosaria Calvello, Davide Bondavalli, Aliana Guerrieri Gonzaga, Monica Marabelli, Bernardo Bonanni, Paolo Radice and the personnel of the Cogentech Cancer Genetic Test Laboratory. The MCCS was made possible by the contribution of many people, including the original investigators, the teams that recruited the participants and continue working on follow-up, and the many thousands of Melbourne residents who continue to participate in the study. We thank the coordinators, the research staff and especially the MMHS participants for their continued collaboration on research studies in breast cancer. MSKCC thanks Marina Corines, Lauren Jacobs. MTLGEBCS would like to thank Martine Tranchant (CHU de Québec - Université Laval Research Center), Marie-France Valois, Annie Turgeon and Lea Heguy (McGill University Health Center, Royal Victoria Hospital; McGill University) for DNA extraction, sample management and skilful technical assistance. J.S. is Chair holder of the Canada Research Chair in Oncogenetics. MYBRCA thanks study participants and research staff (particularly Patsy Ng, Nurhidayu Hassan, Yoon Sook-Yee, Daphne Lee, Lee Sheau Yee, Phuah Sze Yee and Norhashimah Hassan) for their contributions and commitment to this study. NBHS and SBCGS thank study participants and research staff for their contributions and commitment to the studies. For NHS and NHS2 the study protocol was approved by the institutional review boards of the Brigham and Women's Hospital and Harvard T.H. Chan School of Public Health, and those of participating registries as required. We would like to thank the participants and staff of the NHS and NHS2 for their valuable contributions as well as the following state cancer registries for their help: AL, AZ, AR, CA, CO, CT, DE, FL, GA, ID, IL, IN, IA, KY, LA, ME, MD, MA, MI, NE, NH, NJ, NY, NC, ND, OH, OK, OR, PA, RI, SC, TN, TX, VA, WA, WY. The authors assume full responsibility for analyses and interpretation of these data. OFBCR thanks Teresa Selander, Nayana Weerasooriya. ORIGO thanks E. Krol-Warmerdam, and J. Blom for patient accrual, administering questionnaires, and managing clinical information. The LUMC survival data were retrieved from the Leiden hospital-based cancer registry system (ONCDOC) with the help of Dr. J. Molenaar. PBCS thanks Louise Brinton, Mark Sherman, Neonila Szeszenia-Dabrowska, Beata Peplonska, Witold Zatonski, Pei Chao, Michael Stagner. The ethical approval for the POSH study is MREC /00/6/69, UKCRN ID: 1137. We thank staff in the Experimental Cancer Medicine Centre (ECMC) supported Faculty of Medicine Tissue Bank and the Faculty of Medicine DNA Banking resource. PREFACE thanks Sonja Oeser and Silke Landrith. We thank the SEARCH and EPIC teams. SGBCC thanks the participants and research coordinator Ms Tan Siew Li. SKKDKFZS thanks all study participants, clinicians, family doctors, researchers and technicians for their contributions and commitment to this study. We thank the SUCCESS Study teams in Munich, Düsseldorf, Erlangen and Ulm. SZBCS thanks Ewa Putresza. UCIBCS thanks 
Irene Masunaka. UKBGS thanks Breast Cancer Now and the Institute of Cancer Research for support and funding of the Breakthrough Generations Study, and the study participants, study staff, and the doctors, nurses and other health care providers and health information sources who have contributed to the study. We acknowledge NHS funding to the Royal Marsden/ICR NIHR Biomedical Research Centre. We acknowledge funding to the Manchester NIHR Biomedical Research Centre (IS-BRC-1215-20007). We thank the UKOPS participants who donated their samples and data and all the researchers, especially Andy Ryan, Simon Gayther and Ian Jacobs. Finally, we thank the PRACTICAL consortium. J. Liu received a scholarship from the China Scholarship Council (Beijing, China). BCAC is funded by Cancer Research UK [C1287/A16563, C1287/A10118], the European Union's Horizon 2020 Research and Innovation Programme (grant numbers 634935 and 633784 for BRIDGES and B-CAST respectively), and by the European Community's Seventh Framework Programme under grant agreement number 223175 (grant number HEALTH-F2-2009-223175) (COGS). The EU Horizon 2020 Research and Innovation Programme funding source had no role in study design, data collection, data analysis, data interpretation or writing of the report. Genotyping of the OncoArray was funded by the NIH Grant U19 CA148065, and Cancer UK Grant C1287/A16563 and the PERSPECTIVE project supported by the Government of Canada through Genome Canada and the Canadian Institutes of Health Research (grant GPH-129344) and, the Ministère de l'Économie, Science et Innovation du Québec through Genome Québec and the PSRSIIRI-701 grant, and the Quebec Breast Cancer Foundation. Funding for the iCOGS infrastructure came from: the European Community's Seventh Framework Programme under grant agreement $\mathrm{n}^{\circ} 223175$ (HEALTH-F2-2009-223175) (COGS), Cancer Research UK (C1287/A10118, C1287/A10710, C12292/A11174, C1281/A12014, C5047/A8384, C5047/A15007, C5047/A10692, C8197/A16565), the National Institutes of Health (CA128978) and Post-Cancer GWAS initiative (1U19 CA148537, 1U19 CA148065 and 1U19 CA148112 - the GAME-ON initiative), the Department of Defence (W81XWH-10-1-0341), the Canadian Institutes of Health Research (CIHR) for the CIHR Team in Familial Risks of Breast Cancer, and Komen Foundation for the Cure, the Breast Cancer Research Foundation, and the Ovarian Cancer Research Fund. The DRIVE Consortium was funded by U19 CA148065. The Australian Breast Cancer Family Study (ABCFS) was supported by grant UM1 CA164920 from the National Cancer Institute (USA). The content of this manuscript does not necessarily reflect the views or policies of the National Cancer Institute or any of the collaborating centers in the Breast Cancer Family Registry (BCFR), nor does mention of trade names, commercial products, or organizations imply endorsement by the USA Government or the BCFR. The ABCFS was also supported by the National Health and Medical Research Council of Australia, the New South Wales Cancer Council, the Victorian Health Promotion Foundation (Australia) and the Victorian Breast Cancer Research Consortium. J.L.H. is a National Health and Medical Research Council (NHMRC) Senior Principal Research Fellow. M.C.S. is a NHMRC Senior Research Fellow. The ABCS study was supported by the Dutch Cancer Society [grants NKI 2007-3839; 2009 4363]. The Australian Breast Cancer Tissue Bank (ABCTB) was supported by the National Health and Medical Research Council of Australia, The Cancer Institute NSW and the National Breast Cancer Foundation. The ACP study is funded by the Breast Cancer Research Trust, UK, and, KM and AL are supported by the NIHR Manchester Biomedical Research Centre and by the ICEP ("This work was also supported by CRUK [grant number C18281/ A19169]"). The work of the BBCC was partly funded by ELAN-Fond of the University Hospital of Erlangen. The BBCS is funded by Cancer Research UK and Breast Cancer Now and acknowledges NHS funding to the NIHR Biomedical Research Centre, and the National Cancer Research Network (NCRN). The BCEES was funded by the National Health and Medical Research Council, Australia and the Cancer Council Western Australia and acknowledges funding from the National Breast Cancer Foundation (JS). For the BCFR-NY, BCFR-PA, BCFR-UT this work was supported by grant UM1 CA164920 from the National Cancer Institute. The content of this manuscript does not necessarily reflect the views or policies of the National Cancer Institute or any of the collaborating centers in the Breast Cancer Family Registry (BCFR), nor does mention of trade names, commercial products, or organizations imply endorsement by the US Government or the BCFR. The BREast Oncology GAlician Network (BREOGAN) is funded by Acción Estratégica de Salud del Instituto de Salud Carlos III FIS PI12/02125/Cofinanciado FEDER; Acción Estratégica de Salud del Instituto de Salud Carlos III FIS Intrasalud (PI13/01136); Programa Grupos Emergentes, Cancer Genetics Unit, Instituto de Investigacion Biomedica Galicia Sur. Xerencia de Xestion Integrada de Vigo-SERGAS, Instituto de Salud Carlos III, Spain; Grant 10CSA012E, Consellería de Industria Programa Sectorial de Investigación Aplicada, PEME I + D e I + D Suma del Plan Gallego de Investigación, Desarrollo e Innovación Tecnológica de la Consellería de Industria de la Xunta de Galicia, Spain; Grant EC11-192. Fomento de la Investigación Clínica Independiente, Ministerio de Sanidad, Servicios Sociales e Igualdad, Spain; and Grant FEDER-Innterconecta. Ministerio de Economia y Competitividad, Xunta de Galicia, Spain. The BSUCH study was supported by the Dietmar-Hopp Foundation, the Helmholtz Society and the German Cancer Research Center (DKFZ). The CAMA study was funded by Consejo Nacional de Ciencia y Tecnología (CONACyT) (SALUD-2002-C01-7462). Sample collection and processing was funded in part by grants from the National Cancer Institute (NCI R01CA120120 and K24CA169004). CBCS is funded by the Canadian Cancer Society (grant \# 313404) and the Canadian Institutes of Health Research. CCGP is supported by funding from the University of Crete. The CECILE study was supported by Fondation de France, Institut National du Cancer (INCa), Ligue Nationale contre le Cancer, Agence Nationale de Sécurité Sanitaire, de l'Alimentation, de l'Environnement et du Travail (ANSES), Agence Nationale de la Recherche (ANR). The CGPS was supported by the Chief Physician Johan Boserup and Lise Boserup Fund, the Danish Medical Research Council, and Herlev and Gentofte Hospital. COLBCCC is supported by the German Cancer Research Center (DKFZ), Heidelberg, Germany. Diana Torres was in part supported by a postdoctoral fellowship from the Alexander von Humboldt Foundation. The American Cancer Society funds the creation, maintenance, and updating of the CPS-II cohort. The CTS was initially supported by the California Breast Cancer Act of 1993 and the California Breast Cancer Research Fund (contract 97-10500) and is currently funded through the National 
Institutes of Health (R01 CA77398, UM1 CA164917, and U01 CA199277). Collection of cancer incidence data was supported by the California Department of Public Health as part of the statewide cancer reporting program mandated by California Health and Safety Code Section 103885. HAC receives support from the Lon V Smith Foundation (LVS39420). The University of Westminster curates the DietCompLyf database funded by Against Breast Cancer Registered Charity No. 1121258 and the NCRN. The coordination of EPIC is financially supported by the European Commission (DG-SANCO) and the International Agency for Research on Cancer. The national cohorts are supported by: Ligue Contre le Cancer, Institut Gustave Roussy, Mutuelle Générale de l'Education Nationale, Institut National de la Santé et de la Recherche Médicale (INSERM) (France); German Cancer Aid, German Cancer Research Center (DKFZ), Federal Ministry of Education and Research (BMBF) (Germany); the Hellenic Health Foundation, the Stavros Niarchos Foundation (Greece); Associazione Italiana per la Ricerca sul Cancro-AIRC-Italy and National Research Council (Italy); Dutch Ministry of Public Health, Welfare and Sports (VWS), Netherlands Cancer Registry (NKR), LK Research Funds, Dutch Prevention Funds, Dutch ZON (Zorg Onderzoek Nederland), World Cancer Research Fund (WCRF), Statistics Netherlands (The Netherlands); Health Research Fund (FIS), PI13/00061 to Granada, PI13/01162 to EPIC-Murcia, Regional Governments of Andalucía, Asturias, Basque Country, Murcia and Navarra, ISCIII RETIC (RD06/0020) (Spain); Cancer Research UK (14136 to EPIC-Norfolk; C570/A16491 and C8221/A19170 to EPIC-Oxford), Medical Research Council (1000143 to EPIC-Norfolk, MR/M012190/1 to EPIC-Oxford) (United Kingdom). The ESTHER study was supported by a grant from the Baden Württemberg Ministry of Science, Research and Arts. Additional cases were recruited in the context of the VERDI study, which was supported by a grant from the German Cancer Aid (Deutsche Krebshilfe). The GC-HBOC (German Consortium of Hereditary Breast and Ovarian Cancer) is supported by the German Cancer Aid (grant no 110837, coordinator: Rita K. Schmutzler, Cologne). This work was also funded by the European Regional Development Fund and Free State of Saxony, Germany (LIFE - Leipzig Research Centre for Civilization Diseases, project numbers 713-241202, 713-241202, 14505/2470, 14575/2470). The GENICA was funded by the Federal Ministry of Education and Research (BMBF) Germany grants 01KW9975/5, 01KW9976/8, 01KW9977/0 and 01KW0114, the Robert Bosch Foundation, Stuttgart, Deutsches Krebsforschungszentrum (DKFZ), Heidelberg, the Institute for Prevention and Occupational Medicine of the German Social Accident Insurance, Institute of the Ruhr University Bochum (IPA), Bochum, as well as the Department of Internal Medicine, Evangelische Kliniken Bonn gGmbH, Johanniter Krankenhaus, Bonn, Germany. The GEPARSIXTO study was conducted by the German Breast Group GmbH. The GESBC was supported by the Deutsche Krebshilfe e. V. [70492] and the German Cancer Research Center (DKFZ). The HABCS study was supported by the Claudia von Schilling Foundation for Breast Cancer Research, by the Lower Saxonian Cancer Society, and by the Rudolf Bartling Foundation. The HEBCS was financially supported by the Helsinki University Hospital Research Fund, the Finnish Cancer Society, and the Sigrid Juselius Foundation. The HERPACC was supported by MEXT Kakenhi (No. 170150181 and 26253041) from the Ministry of Education, Science, Sports, Culture and Technology of Japan, by a Grant-in-Aid for the Third Term Comprehensive 10-Year Strategy for Cancer Control from Ministry Health, Labour and Welfare of Japan, by Health and Labour Sciences Research Grants for Research on Applying Health Technology from Ministry Health, Labour and Welfare of Japan, by National Cancer Center Research and Development Fund, and "Practical Research for Innovative Cancer Control (15ck0106177h0001)" from Japan Agency for Medical Research and development, AMED, and Cancer Bio Bank Aichi. The HMBCS was supported by a grant from the Friends of Hannover Medical School and by the Rudolf Bartling Foundation. The HUBCS was supported by a grant from the German Federal Ministry of Research and Education (RUS08/017), B.M. was supported by grant 17-44-020498, 17-29-06014 of the Russian Foundation for Basic Research, D.P. was supported by grant 18-29-09129 of the Russian Foundation for Basic Research, E.K was supported by the program for support the bioresource collections №007-030164/2, and the study was performed as part of the assignment of the Ministry of Science and Higher Education of the Russian Federation (№AAAA-A16-116020350032-1). Financial support for KARBAC was provided through the regional agreement on medical training and clinical research (ALF) between Stockholm County Council and Karolinska Institutet, the Swedish Cancer Society, The Gustav V Jubilee foundation and Bert von Kantzows foundation. The KARMA study was supported by Märit and Hans Rausings Initiative Against Breast Cancer. The KBCP was financially supported by the special Government Funding (EVO) of Kuopio University Hospital grants, Cancer Fund of North Savo, the Finnish Cancer Organizations, and by the strategic funding of the University of Eastern Finland. The KOHBRA study was partially supported by a grant from the Korea Health Technology R\&D Project through the Korea Health Industry Development Institute (KHIDI), and the National R\&D Program for Cancer Control, Ministry of Health \& Welfare, Republic of Korea (HI16C1127; 1020350; 1420190). LMBC is supported by the 'Stichting tegen Kanker'. DL is supported by the FWO. The MABCS study is funded by the Research Centre for Genetic Engineering and Biotechnology "Georgi D. Efremov", MASA. The MARIE study was supported by the Deutsche Krebshilfe e.V. [70-2892-BR I, 106332, 108253, 108419, 110826, 110828], the Hamburg Cancer Society, the German Cancer Research Center (DKFZ) and the Federal Ministry of Education and Research (BMBF) Germany [01KH0402]. MBCSG is supported by grants from the Italian Association for Cancer Research (AIRC) and by funds from the Italian citizens who allocated the 5/1000 share of their tax payment in support of the Fondazione IRCCS Istituto Nazionale Tumori, according to Italian laws (INT-Institutional strategic projects “ $5 \times 1000$ ”). The MCBCS was supported by the NIH grants CA192393, CA116167, CA176785 an NIH Specialized Program of Research Excellence (SPORE) in Breast Cancer [CA116201], and the Breast Cancer Research Foundation and a generous gift from the David F. and Margaret T. Grohne Family Foundation. The Melbourne Collaborative Cohort Study (MCCS) cohort recruitment was funded by VicHealth and Cancer Council Victoria. The MCCS was further augmented by Australian National Health and Medical Research Council grants 209057, 396414 and 1074383 and by infrastructure provided by Cancer Council Victoria. Cases and their vital status were ascertained through the Victorian Cancer Registry and the Australian Institute of Health and Welfare, including the National Death Index 
and the Australian Cancer Database. The MEC was supported by NIH grants CA63464, CA54281, CA098758, CA132839 and CA164973. The MISS study is supported by funding from ERC-2011-294576 Advanced grant, Swedish Cancer Society, Swedish Research Council, Local hospital funds, Berta Kamprad Foundation, Gunnar Nilsson. The MMHS study was supported by NIH grants CA97396, CA128931, CA116201, CA140286 and CA177150. MSKCC is supported by grants from the Breast Cancer Research Foundation and Robert and Kate Niehaus Clinical Cancer Genetics Initiative. The work of MTLGEBCS was supported by the Quebec Breast Cancer Foundation, the Canadian Institutes of Health Research for the "CIHR Team in Familial Risks of Breast Cancer" program - grant \# CRN-87521 and the Ministry of Economic Development, Innovation and Export Trade - grant \# PSR-SIIRI-701. MYBRCA is funded by research grants from the Malaysian Ministry of Higher Education (UM.C/HlR/MOHE/06) and Cancer Research Malaysia. The NBCS has received funding from the K.G. Jebsen Centre for Breast Cancer Research; the Research Council of Norway grant 193387/V50 (to A-L Børresen-Dale and V.N. Kristensen) and grant 193387/H10 (to A-L Børresen-Dale and V.N. Kristensen), South Eastern Norway Health Authority (grant 39346 to A-L Børresen-Dale) and the Norwegian Cancer Society (to A-L Børresen-Dale and V.N. Kristensen). The NBHS was supported by NIH grant R01CA100374. Biological sample preparation was conducted the Survey and Biospecimen Shared Resource, which is supported by P30 CA68485. The Northern California Breast Cancer Family Registry (NC-BCFR) and Ontario Familial Breast Cancer Registry (OFBCR) were supported by grant UM1 CA164920 from the National Cancer Institute (USA). The content of this manuscript does not necessarily reflect the views or policies of the National Cancer Institute or any of the collaborating centers in the Breast Cancer Family Registry (BCFR), nor does mention of trade names, commercial products, or organizations imply endorsement by the USA Government or the BCFR. The Carolina Breast Cancer Study was funded by Komen Foundation, the National Cancer Institute (P50 CA058223, U54 CA156733, U01 CA179715), and the North Carolina University Cancer Research Fund. The NGOBCS was supported by the National Cancer Center Research and Development Fund (Japan). The NHS was supported by NIH grants P01 CA87969, UM1 CA186107, and U19 CA148065. The NHS2 was supported by NIH grants UM1 CA176726 and U19 CA148065. The ORIGO study was supported by the Dutch Cancer Society (RUL 1997-1505) and the Biobanking and Biomolecular Resources Research Infrastructure (BBMRI-NL CP16). The PBCS was funded by Intramural Research Funds of the National Cancer Institute, Department of Health and Human Services, USA. Genotyping for PLCO was supported by the Intramural Research Program of the National Institutes of Health, NCI, Division of Cancer Epidemiology and Genetics. The PLCO is supported by the Intramural Research Program of the Division of Cancer Epidemiology and Genetics and supported by contracts from the Division of Cancer Prevention, National Cancer Institute, National Institutes of Health. The POSH study is funded by Cancer Research UK (grants C1275/A11699, C1275/C22524, C1275/A19187, C1275/A15956 and Breast Cancer Campaign 2010PR62, 2013PR044. The SBCGS was supported primarily by NIH grants R01CA64277, R01CA148667, UMCA182910, and R37CA70867. Biological sample preparation was conducted the Survey and Biospecimen Shared Resource, which is supported by P30 CA68485. The scientific development and funding of this project were, in part, supported by the Genetic Associations and Mechanisms in Oncology (GAME-ON) Network U19 CA148065. SEARCH is funded by Cancer Research UK [C490/A10124, C490/A16561] and supported by the UK National Institute for Health Research Biomedical Research Centre at the University of Cambridge. The University of Cambridge has received salary support for PDPP from the NHS in the East of England through the Clinical Academic Reserve. SEBCS was supported by the BRL (Basic Research Laboratory) program through the National Research Foundation of Korea funded by the Ministry of Education, Science and Technology (2012-0000347). SGBCC is funded by the National Research Foundation Singapore, NUS start-up Grant, National University Cancer Institute Singapore (NCIS) Centre Grant, Breast Cancer Prevention Programme, Asian Breast Cancer Research Fund and the NMRC Clinician Scientist Award. Additional controls were recruited by the Singapore Consortium of Cohort Studies-Multi-ethnic cohort (SCCS-MEC), which was funded by the Biomedical Research Council, grant number: 05/1/21/19/425. The Sister Study (SISTER) is supported by the Intramural Research Program of the NIH, National Institute of Environmental Health Sciences (Z01-ES044005 and Z01-ES049033). The Two Sister Study (2SISTER) was supported by the Intramural Research Program of the NIH, National Institute of Environmental Health Sciences (Z01-ES044005 and Z01-ES102245), and, also by a grant from Susan G. Komen for the Cure, grant FAS0703856. SKKDKFZS is supported by the DKFZ. The SMC is funded by the Swedish Cancer Foundation and the Swedish Research Council (VR 201700644) grant for the Swedish Infrastructure for Medical Population-based Life-course Environmental Research (SIMPLER). The SZBCS was supported by Grant PBZ_KBN_122/P05/2004 and the program of the Minister of Science and Higher Education under the name "Regional Initiative of Excellence" in 2019-2022 project number 002/RID/2018/19 amount of financing 12000000 PLN. The TWBCS is supported by the Taiwan Biobank project of the Institute of Biomedical Sciences, Academia Sinica, Taiwan. Ascertainment and data collection for the UBCS is supported by funding from National Cancer Institute grants R01 CA163353 (to N.J. Camp) and the Women's Cancer Center at the Huntsman Cancer Institute (HCI) which is funded in part by the Huntsman Cancer Foundation. Data collection is also made possible by the Utah Population Database (UPDB) and the Utah Cancer Registry (UCR). Support for the UPDB is provided by the University of Utah, HCI, and the Comprehensive Cancer Center Support grant NCI P30 CA42014. The UCR is funded by the NCI's SEER Program, Contract No. HHSN261201800016I, with additional support from the US Center for Disease Control and Prevention's National Program of Cancer Registries, Cooperative Agreement No. NU58DP0063200, the University of Utah and Huntsman Cancer Foundation. The UCIBCS component of this research was supported by the NIH [CA58860, CA92044] and the Lon V Smith Foundation [LVS39420]. The UKBGS is funded by Breast Cancer Now and the Institute of Cancer Research (ICR), London. ICR acknowledges NHS funding to the NIHR Biomedical Research Centre. The UKOPS study was funded by The Eve Appeal (The Oak Foundation) and supported by the National Institute for Health Research University College London Hospitals Biomedical 
Research Centre. The WAABCS study was supported by grants from the National Cancer Institute of the National Institutes of Health (R01 CA89085 and P50 CA125183 and the D43 TW009112 grant), Susan G. Komen (SAC110026), the Dr. Ralph and Marian Falk Medical Research Trust, and the Avon Foundation for Women. The Prostate cancer genome-wide association analyses are supported by the Canadian Institutes of Health Research, European Commission's Seventh Framework Programme grant agreement n ${ }^{\circ} 223175$ (HEALTH-F2-2009-223175), Cancer Research UK Grants C5047/A7357, C1287/A10118, C1287/A16563, C5047/A3354, C5047/A10692, C16913/A6135, and The National Institute of Health (NIH) Cancer Post-Cancer GWAS initiative grant: No. 1 U19 CA 148537-01 (the GAME-ON initiative). Genotyping of the OncoArray was funded by the US National Institutes of Health (NIH) [U19 CA 148537 for ELucidating Loci Involved in Prostate cancer SuscEptibility (ELLIPSE) project and X01HG007492 to the Center for Inherited Disease Research (CIDR) under contract number HHSN268201200008I] and by Cancer Research UK grant A8197/A16565. Additional analytic support was provided by NIH NCI U01 CA188392 (PI: Schumacher). We would also like to thank the following for funding support: The Institute of Cancer Research and The Everyman Campaign, The Prostate Cancer Research Foundation, Prostate Research Campaign UK (now PCUK), The Orchid Cancer Appeal, Rosetrees Trust, The National Cancer Research Network UK, The National Cancer Research Institute (NCRI) UK. We are grateful for support of NIHR funding to the NIHR Biomedical Research Centre at The Institute of Cancer Research and The Royal Marsden NHS Foundation Trust.

\title{
Author contributions
}

J. Liu analyzed the data and wrote, revised and approved the manuscript. W.J.C.P-vdS. analyzed the data, read and approved the manuscript. J.M.C. designed the study, contributed samples, provided clinical data and revised and approved the manuscript. M.K.B. and Q.W. provided database management, contributed samples, provided clinical data and read and approved the manuscript. K.M and J.D. analyzed the data, contributed samples, provided clinical data and read and approved the manuscript. T.U.A., K.A., C.B.A., I.A., H.A-C., N.N.A., V.A., N.A., K.J.A., A.A., P.A., H. Becher, M.W.B., S.B., M.B., L.B., N.V.B., N.B-M., S.E.B., H. Brauch, H. Brenner, I.B., S.Y.B., T.B., B.B., Q.C., H.C., D.C., F.C., J.E.C., J.C-C., S.J.C., J-Y.C., M.C., C.L.C., NBCS Collaborators, F.J.C., K.C., M.B.D., P.D., I.d-S-S., M.D., D.M.E., A.H.E., P.A.F., J.F., H.F., L.F., M.G-D., S.M.G., M.G-C., J.A.G-S., M.M.G., G.G.G., M.S.G., D.E.G., P.G., C.A.H., N.H., P. Hall, P.A.H., S.N.H., M.H., P. Hillemans, J.L.H., M-F.H., D.J.H., D.H., ABCTB Investigators, H.I., M.I., M.J., A.J., E.M.J., R. Kaaks, D.K., R. Keeman., E.K., S-W.K., P.K., V.N.K., A.W.K., L.L.M., J.Li, A. Lindblom., A. Lophatananon., R.N.L., J. Lubinski, A. Mannermaa, M.M., S. Manoukian, S. Margolin, S. Mariapun, K. Matsuo, T.M., D.M., A. Meindl, U.M., R.L.M., K.Muir, A.M.M., S.L.N., H.N., K.O., O.I.O., J.E.O., H.O., N.O., S.K.P., P.P., J.P., D.P-K., N.P., B.R., R.R-M., G.R., H.S.R., V.R., A.R., M.R., E.S., R.K.S., A.S., C.Scott, M.S., C-Y.S., X-O.S., J.S., C.Sohn, M.C.S., J.J.S., R.M.T., W.J.T., S.H.T., M.T., D.T., T.T., M.U., C.M.V., C.J.vA., A.W., T.Y., W.Z., A.Z. and E.Z. contributed samples, provided clinical data and read and approved the manuscript. G.T-M., T.D., A.J.S., U.H. and M.K.S. contributed samples, provided clinical data and revised and approved the manuscript. A.M.D., P.D.P.P. and D.F.E. designed the study, contributed samples, provided clinical data and funding and read and approved the manuscript. M.J.H. designed the study, contributed samples, provided clinical data and revised and approved the manuscript. J.W.M.M. designed the study, read and approved the manuscript and provided funding. A.H. conceived of the study, designed the study, contributed samples and clinical data, analyzed the data and wrote, revised and approved the manuscript.

\section{Competing interests}

Matthias W. Beckmann conducts research funded by Amgen, Novartis and Pfizer. Peter A. Fasching conducts research funded by Amgen, Novartis and Pfizer. He received Honoraria from Roche, Novartis and Pfizer. Allison W. Kurian received research funding to her institution from Myriad Genetics for an unrelated project (funding dates 2017-2019). Usha Menon has stocks in Abcodia awarded to her by UCL. Paolo Peterlongo is a member of the Editorial Board of Scientific Reports. All other authors declare no conflict of interest.

\section{Additional information}

Supplementary information is available for this paper at https://doi.org/10.1038/s41598-020-65665-y.

Correspondence and requests for materials should be addressed to A.H.

Reprints and permissions information is available at www.nature.com/reprints.

Publisher's note Springer Nature remains neutral with regard to jurisdictional claims in published maps and institutional affiliations.

\begin{abstract}
Open Access This article is licensed under a Creative Commons Attribution 4.0 International format, as long as you give appropriate credit to the original author(s) and the source, provide a link to the Creative Commons license, and indicate if changes were made. The images or other third party material in this article are included in the article's Creative Commons license, unless indicated otherwise in a credit line to the material. If material is not included in the article's Creative Commons license and your intended use is not permitted by statutory regulation or exceeds the permitted use, you will need to obtain permission directly from the copyright holder. To view a copy of this license, visit http://creativecommons.org/licenses/by/4.0/.
\end{abstract}

(c) The Author(s) 2020 


\section{NBCS Collaborators}

Kristine K. Sahlberg ${ }^{53,55}$, Anne-Lise Børresen-Dale ${ }^{53,54}$, Lars Ottestad ${ }^{53}$, Rolf Kåresen ${ }^{54,56}$, Ellen Schlichting ${ }^{56}$, Marit Muri Holmen ${ }^{57}$, Toril Sauer ${ }^{58}$, Vilde Haakensen ${ }^{53}$, Olav Engebråten ${ }^{54,59}$, Bjørn Naume ${ }^{60}$, Alexander Fosså ${ }^{60}$, Cecile E. Kiserud ${ }^{61}$, Kristin V. Reinertsen ${ }^{61}$, Åslaug Helland ${ }^{53,60}$, Margit Riis ${ }^{53}$, Jürgen Geisler ${ }^{62}$, OSBREAC* \& Grethe I. Grenaker Alnæs ${ }^{53}$

${ }^{53}$ Department of Cancer Genetics, Institute for Cancer Research, Oslo University Hospital-Radiumhospitalet, Oslo, Norway. ${ }^{54}$ Institute of Clinical Medicine, Faculty of Medicine, University of Oslo, Oslo, Norway. ${ }^{55}$ Department of Research, Vestre Viken Hospital, Drammen, Norway. ${ }^{56}$ Section for Breast- and Endocrine Surgery, Department of Cancer, Division of Surgery, Cancer and Transplantation Medicine, Oslo University Hospital-Ullevål, Oslo, Norway. ${ }^{57}$ Department of Radiology and Nuclear Medicine, Oslo University Hospital, Oslo, Norway. ${ }^{58}$ Department of Pathology, Akershus University Hospital, Lørenskog, Norway. ${ }^{59} 59$ Department of Tumor Biology, Institute for Cancer Research, Oslo University Hospital, Oslo, Norway. ${ }^{60}$ Department of Oncology, Division of Surgery, Cancer and Transplantation Medicine, Oslo University Hospital-Radiumhospitalet, Oslo, Norway. ${ }^{61}$ National Advisory Unit on Late Effects after Cancer Treatment, Oslo University Hospital-Radiumhospitalet, Oslo, Norway. ${ }^{62}$ Department of Oncology, Akershus University Hospital, Lørenskog, Norway.

\section{OSBREAC}

Tone F. Bathen ${ }^{176}$, Elin Borgen ${ }^{177}$, Britt Fritzman ${ }^{178}$, Øystein Garred ${ }^{177}$, Gry Aarum Geitvik ${ }^{53}$, Solveig Hofvind ${ }^{179,180}$, Anita Langerød ${ }^{53}$, Ole Christian Lingjærde ${ }^{181,182}$, Gunhild Mari Mælandsmo ${ }^{59,183}$, Hege G Russnes ${ }^{53,177}$, Helle Kristine Skjerven ${ }^{184}$ \& Therese Sørlie $^{53}$

${ }^{176}$ Department of Circulation and Medical Imaging, Norwegian University of Science and Technology (NTNU), Trondheim, Norway. ${ }^{177}$ Department of Pathology, Oslo University Hospital, Oslo, Norway. ${ }^{178} \oslash$ stfold Hospital, Østfold, Norway. ${ }^{179}$ Cancer Registry of Norway, Oslo, Oslo, Norway. ${ }^{180}$ Akershus University College of Applied Sciences, Faculty of Health Science, Oslo, Norway. ${ }^{181}$ Centre for Cancer Biomedicine, University of Oslo, Oslo, Norway. ${ }^{182}$ Department of Computer Science, University of Oslo, Oslo, Norway. ${ }^{183}$ Department of Pharmacy, Faculty of Health Sciences, University of Tromsø, Tromsø, Norway. ${ }^{184}$ Breast and Endocrine Surgery, Department of Breast and Endocrine Surgery, Vestre Viken Hospital, Drammen, Norway.

\section{ABCTB Investigators}

Christine Clarke ${ }^{163}$, Deborah Marsh ${ }^{164}$, Rodney Scott ${ }^{165,166}$, Robert Baxter ${ }^{167}$, Desmond Yip $^{168,169}$, Jane Carpenter ${ }^{170}$, Alison Davis ${ }^{171,172}$, Nirmala Pathmanathan ${ }^{173,174}$, Peter Simpson $^{175}$, Dinny Graham ${ }^{163}$ \& Mythily Sachchithananthan ${ }^{163}$

${ }^{163}$ Centre for Cancer Research, The Westmead Institute for Medical Research, The University of Sydney, Sydney, New South Wales, Australia. ${ }^{164}$ Translational Oncology Group, School of Life Sciences, Faculty of Science, University of Technology Sydney, Sydney, New South Wales, Australia. ${ }^{165}$ School of Biomedical Sciences, University of Newcastle, Newcastle, UK. ${ }^{166}$ Hunter Medical Research Institute and NSW Health Pathology North, Newcastle, Australia. ${ }^{167}$ Kolling Institute of Medical Research, University of Sydney, Sydney, New South Wales, Australia. ${ }^{168}$ Epigenetics and Transcription Laboratory, Melanie Swan Memorial Translational Centre, Sci-Tech, University of Canberra, Canberra, Australian Capital Territory, Australia. ${ }^{169}$ Department of Medical Oncology, The Canberra Hospital, Canberra, Australia. ${ }^{170}$ Scientific Platforms, The Westmead Institute for Medical Research, University of Sydney, Sydney, New South Wales, Australia. ${ }^{171}$ The Canberra Hospital, Canberra, Australia. ${ }^{172}$ The Australian National University, Canberra, Australia. ${ }^{173}$ Westmead Breast Cancer Institute, Western Sydney Local Health District, Sydney, New South Wales, Australia. ${ }^{174}$ University of Sydney, Western Clinical School, Sydney, New South Wales, Australia. ${ }^{175} \mathrm{UQ}$ Centre for Clinical Research, Faculty of Medicine, The University of Queensland, Brisbane, Australia. 